\title{
A "Liquid-Solid" Phase Transition in a Simple Model for Swarming, Based on the "No Flat-Spots" Theorem for Subharmonic Functions
}

\author{
Rupert L. Frank \& ELLIOTT H. LieB
}

\begin{abstract}
We consider a family of non-local shape optimization problems, which are motivated by a simple model for swarming and other self-assembly/aggregation models, and prove the existence of different phases for several of them. A technical key ingredient, which we establish, is that a strictly subharmonic function cannot be constant on a set of positive measure.
\end{abstract}

\section{InTRODUCTION AND MAIN RESUlT}

We are interested in the following minimization problem, depending on a parameter $\alpha>0$, which was recently introduced by Burchard, Choksi, and Topaloglu in [5]. For measurable functions $\rho \geq 0$ on $\mathbb{R}^{3}$, one sets

$$
\mathcal{E}_{\alpha}[\rho]=\frac{1}{2} \iint_{\mathbb{R}^{3} \times \mathbb{R}^{3}} \rho(x)\left(\frac{1}{|x-y|}+|x-y|^{\alpha}\right) \rho(y) \mathrm{d} x \mathrm{~d} y
$$

and, for $m>0$,

$$
E_{\alpha}(m)=\inf \left\{\mathcal{E}_{\alpha}[\rho]: 0 \leq \rho \leq 1 \int_{\mathbb{R}^{3}} \rho(x) \mathrm{d} x=m\right\} .
$$

This is a simple model problem for flocking of birds or some other condensation phenomenon. The function $\rho$ describes the density of birds (or "particles"). The energy functional $\mathcal{E}_{\alpha}[\rho]$ has two terms. The first, $|x-y|^{-1}$, is a two-body repulsive interaction between pairs of birds or particles. The second, $|x-y|^{\alpha}$, is a two-body attractive interaction that engenders condensation (or "flocking"). 
The condition that $\rho \leq 1$, introduced in [5], is a many-body hard-core repulsion at short range. It imposes a maximum density, beyond which the birds would be crushed. Its analogy in statistical physics is a bound on the allowed density of atoms in a liquid, namely the density of the solid state. (For background on mathematical models for biological aggregations, see, for instance, $[2,4,12]$ and references therein.)

We know from [9] that for any $\alpha>0$ and $m>0$, the problem $E_{\alpha}(m)$ has a minimizer.

It is natural to think of the following three phases of the model, as described by the level set $\{\rho=1\}$ of a minimizer $\rho$ of the $E_{\alpha}(m)$ problem:

$$
\begin{array}{ll}
\text { Phase 1: } & |\{\rho=1\}|=0, \\
\text { Phase 2: } & 0<|\{\rho=1\}|<m, \\
\text { Phase 3: } & |\{\rho=1\}|=m .
\end{array}
$$

(Since we do not know whether minimizers are unique (modulo translations), it is possible to have mixtures of these phases.) We think of phase 1 as a "liquid phase," phase 3 as a "solid phase," and phase 2 as an "intermediate phase."

The following theorems establish rigorously the existence of phases 1 and 3 .

Theorem 1.1. For any $\alpha>0$ there exists an $m_{c_{1}}(\alpha)>0$ such that, for $m<m_{c_{1}}(\alpha)$, any minimizer $\rho$ for $E_{\alpha}(m)$ satisfies $|\{\rho=1\}|=0$.

Theorem 1.2. For any $\alpha>0$ there exists an $m_{c_{2}}(\alpha)<\infty$ such that, for $m>m_{c_{2}}(\alpha)$, any minimizer for $E_{\alpha}(m)$ satisfies $|\{\rho=1\}|=m$.

Our results do not establish the existence of an intermediate phase 2 . In the exactly solvable case $\alpha=2$ [5], it is shown that phase 2 does not occur, but we believe this is an untypical behavior. (This belief is supported by the candidates from [11] for minimizers for $E_{\alpha}(m)$ when $m$ is small and $\alpha \neq 2$, which are not characteristic functions. Note that the computations in [11] do not impose the constraint $\rho \leq 1$. Therefore, by the arguments in the proof of Lemma 5.2 below, they are relevant for our problem for small $m$.)

Results similar to ours were obtained in [5] for kernels of the form $|x|^{-p}+|x|^{2}$ with $1<p<3$ in the analogue of Theorem 1.1 and $0<p<3$ in the analogue of Theorem 1.2. The proofs of these results, however, rely heavily on the algebraic properties of $|x|^{2}$. It is conjectured in [5] that these qualitative facts should be true for a larger class of interaction kernels, and our results confirm this expectation in another class of kernels.

Our results are not restricted to Coulomb singularities $|x|^{-1}$. For example, we can extend Theorem 1.2 to the case of interaction kernels of the form

$$
|x|^{-\beta}+|x|^{\alpha} \quad \text { with } 0<\beta<1 \text { and } \alpha>0 .
$$

We explain this in Subsection 5.4. Neither for this extension nor for our main results do we need the kernels to have an exact power law behavior, but we prefer to stick to this model case in order to make the arguments as simple as possible. 
We end this introduction with the discussion of a related shape optimization problem, which was the main focus of the work of Burchard, Choksi, and Topaloglu in [5]. For $m>0$, we set

$$
I_{\alpha}(m)=\inf \left\{\mathcal{E}_{\alpha}\left[\chi_{\Omega}\right]: \Omega \subset \mathbb{R}^{3},|\Omega|=m\right\} .
$$

As a consequence of Theorems 1.1 and 1.2, we obtain the existence of a phase transition with respect to the parameter $m$.

Corollary 1.3. For any $\alpha>0$, there are $0<\tilde{m}_{c_{1}}(\alpha) \leq \tilde{m}_{c_{2}}(\alpha)<\infty$ such that $I_{\alpha}(m)$ has a minimizer for $m>\tilde{m}_{c_{2}}(\alpha)$ and has no minimizer for $m<\tilde{m}_{c_{1}}(\alpha)$.

It is natural to conjecture that $\tilde{m}_{c_{1}}(\alpha)=\tilde{m}_{c_{2}}(\alpha)$, and to wonder whether minimizers, whenever they exist, are spherically symmetric. These properties are true for $\alpha=2$, where the model is explicitly solvable [5]. The following proof gives $m_{c_{1}}(\alpha) \leq \tilde{m}_{c_{1}}(\alpha)$ and $\tilde{m}_{c_{2}}(\alpha) \leq m_{c_{2}}(\alpha)$. (The strict inequality $m_{c_{1}}(\alpha)<\tilde{m}_{c_{1}}(\alpha)$ occurs if for some $m \in\left(m_{c_{1}}(\alpha), \tilde{m}_{c_{1}}(\alpha)\right)$ all minimizers $\rho$ of the problem $E_{\alpha}(m)$ satisfy $|\{0<\rho<1\}|>0$. Similarly, the strict inequality $\tilde{m}_{c_{2}}(\alpha)<m_{c_{2}}(\alpha)$ would occur if for some $m \in\left(\tilde{m}_{c_{2}}(\alpha), m_{c_{2}}(\alpha)\right)$ the problem $E_{\alpha}(m)$ had both a minimizer which is a characteristic function and one that is not.)

Proof of Corollary 1.3. We clearly have $E_{\alpha}(m) \leq I_{\alpha}(m)$. The important observation from [5] is that, in fact,

$$
E_{\alpha}(m)=I_{\alpha}(m) .
$$

Moreover, the problem $I_{\alpha}(m)$ has a minimizer if and only if the characteristic function of a set is a minimizer for the relaxed problem $E_{\alpha}(m)$. Thus, according to Theorem 1.2, the $I_{\alpha}(m)$ problem has a minimizer for $m>m_{c_{2}}(\alpha)$ and, according to Theorem 1.1, has no minimizer for $m<m_{c_{1}}(\alpha)$.

\section{Weak Derivatives on SETS of CONSTANCy}

The following result about functions in Sobolev spaces will play an important role in our proof.

Proposition 2.1. Let $\Omega \subset \mathbb{R}^{d}$ be an open set, $k \in \mathbb{N}$ and $u \in W_{\text {loc }}^{k, 1}(\Omega)$ realvalued. Then, for any Borel set $A \subset \mathbb{R}$ of zero measure and any multi-index $\alpha \in \mathbb{N}_{0}^{d}$ with $0<|\alpha| \leq k$,

$$
\partial^{\alpha} u=0 \quad \text { almost everywhere on } u^{-1}(A) .
$$

Proof. For $k=1$, this is a classical result $[1,17,19]$ (see also [15, Theorem 6.19] for a textbook proof), and we now show that this implies the general result by a simple induction argument. Thus, let $k \geq 2$ and $0<|\alpha| \leq k$, and write $\partial^{\alpha}=\partial_{j} \partial^{\beta}$ for some $1 \leq j \leq d$ and some multi-index $\beta$ with $0 \leq|\beta| \leq k-1$. 
By induction, we have $v:=\partial^{\beta} u=0$ almost everywhere on $u^{-1}(A)$, so we have $v^{-1}(\{0\}) \supset u^{-1}(A)$ (up to sets of measure zero). Moreover, since $v \in W_{\text {loc }}^{1,1}(\Omega)$, we have again by the Almgren-Lieb result that $\partial_{j} v=0$ almost everywhere on $v^{-1}(\{0\})$. In particular, $\partial_{j} v=0$ almost everywhere on $u^{-1}(A)$, which proves the assertion.

From this proposition we deduce, in particular, that $\Delta u=0$ almost everywhere on $\{u=\tau\}$, which leads immediately to the following interesting corollary about strictly subharmonic functions.

Corollary 2.2 (Strictly subharmonic functions have no flat spots). We let $\Omega \subset \mathbb{R}^{d}, d \geq 2$, be an open set, and assume that $u \in W_{\mathrm{loc}}^{2,1}(\Omega)$ satisfies $-\Delta u \leq-\varepsilon$ in $\Omega$ for some $\varepsilon>0$. Then, $|\{x \in \Omega: u(x)=\tau\}|=0$ for any $\tau \in \mathbb{R}$.

The need of some strict subharmonicity assumption to deduce the absence of flat spots can be seen from the example $u(x)=\left(x_{1}\right)_{+}$, which is subharmonic and constant on the half-space $\left\{x_{1} \leq 0\right\}$.

The conclusion of the corollary remains valid if the assumption $u \in W_{\text {loc }}^{2,1}(\Omega)$ is replaced by continuity and the equation $-\Delta u \leq-\varepsilon$ is understood in the viscosity sense. In fact, it remains valid for $u \in L_{\text {loc }}^{1}(\Omega)$ under the assumption that the Lebesgue measure is absolutely continuous with respect to the measure $\Delta u$. We will not need these results but, since they might be of independent interest, we present their proofs in two appendices.

\section{The EUler-Lagrange EQUATION AND THE "ChEMiCAl POTENTIAL"}

In order to emphasize the general nature of the arguments in this section, we consider more general interaction kernels $k$ that are locally integrable, non-negative, and lower semi-continuous, and which satisfy $\lim _{|x| \rightarrow \infty} k(x)=\infty$. We set

$$
\mathcal{E}[\rho]=\frac{1}{2} \iint_{\mathbb{R}^{3} \times \mathbb{R}^{3}} \rho(x) k(x-y) \rho(y) \mathrm{d} x \mathrm{~d} y
$$

and

$$
E(m)=\inf \left\{\mathcal{E}[\rho]: 0 \leq \rho \leq 1, \int_{\mathbb{R}^{3}} \rho(x) \mathrm{d} x=m\right\} .
$$

Existence of minimizers has been proved under the above conditions on $k$ in [7, 20] for the problem without the $L^{\infty}$ constraint. The case of the $L^{\infty}$ constraint is, in fact, simpler. Moreover, the assumption of spherical symmetry of $k$ in [20] is not necessary.

Let $\rho$ be a minimizer for $E(m)$, and let

$$
\varphi(x)=\int_{\mathbb{R}^{3}} k(x-y) \rho(y) \mathrm{d} y
$$


be its potential. In [5] it is shown that there is a constant $\mu>0$ such that, for almost every $x \in \mathbb{R}^{3}$,

$$
\varphi(x) \begin{cases}\leq \mu & \text { if } \rho(x)=1, \\ =\mu & \text { if } 0<\rho(x)<1, \\ \geq \mu & \text { if } \rho(x)=0 .\end{cases}
$$

We now identify $\mu$ with the "chemical potential" (i.e., the derivative of $E$ with respect to $m$ ) of the minimization problem.

Lemma 3.1. Let $\rho$ be a minimizer of $E(m)$ for some $m>0$, and let $\mu$ be as in (3.4). Then,

$$
\limsup _{m^{\prime} \downarrow m} \frac{E\left(m^{\prime}\right)-E(m)}{m^{\prime}-m} \leq \mu \leq \liminf _{m^{\prime} \uparrow m} \frac{E\left(m^{\prime}\right)-E(m)}{m^{\prime}-m} .
$$

Note that this implies, in particular, that $E$ is a continuous function of $m$ and that the singular part of its distributional derivative is non-positive.

Proof. Let $\varepsilon>0$. Since the potential $\varphi$ is continuous (see, e.g., the remark before [5, Theorem 4.4]), the set $F_{\varepsilon}=\{\mu<\varphi \leq \mu+\varepsilon\}$ has positive measure. Since $\lim _{|x| \rightarrow \infty} \varphi(x)=\infty$ (see, e.g., the proof of Lemma 4.3 in [5]), the set $F_{\varepsilon}$ is bounded. Note that according to (3.4), $\rho=0$ on $F_{\varepsilon}$. For any $m<m^{\prime} \leq m+\left|F_{\varepsilon}\right|$, we consider $\tilde{\rho}:=\rho+\left(\left(m^{\prime}-m\right) /\left|F_{\varepsilon}\right|\right) \chi_{F_{\varepsilon}}$, which clearly satisfies the constraints of the $E\left(m^{\prime}\right)$ problem. Moreover,

$$
\begin{aligned}
& E\left(m^{\prime}\right) \leq \mathcal{E}[\tilde{\rho}] \\
= & \mathcal{E}[\rho]+\frac{m^{\prime}-m}{\left|F_{\varepsilon}\right|} \iint_{\mathbb{R}^{3} \times \mathbb{R}^{3}} \chi_{F_{\varepsilon}}(x) k(x-y) \rho(y) \mathrm{d} x \mathrm{~d} y+\left(\frac{m^{\prime}-m}{\left|F_{\varepsilon}\right|}\right)^{2} \mathcal{E}\left[\chi_{F_{\varepsilon}}\right] \\
= & E(m)+\frac{m^{\prime}-m}{\left|F_{\varepsilon}\right|} \int_{F_{\varepsilon}} \varphi(x) \mathrm{d} x+\left(\frac{m^{\prime}-m}{\left|F_{\varepsilon}\right|}\right)^{2} \mathcal{E}\left[\chi_{F_{\varepsilon}}\right] \\
\leq & E(m)+(\mu+\varepsilon)\left(m^{\prime}-m\right)+\left(\frac{m^{\prime}-m}{\left|F_{\varepsilon}\right|}\right)^{2} \mathcal{E}\left[\chi_{F_{\varepsilon}}\right] .
\end{aligned}
$$

Letting $m^{\prime} \downarrow m$, we find

$$
\limsup _{m^{\prime} \downarrow m} \frac{E\left(m^{\prime}\right)-E(m)}{m^{\prime}-m} \leq \mu+\varepsilon
$$

and, since $\varepsilon>0$ is arbitrary, we obtain the left inequality in the lemma.

To prove the reverse inequality, we distinguish two cases. First, assume that $\inf \varphi<\mu$. Then, for fixed $\varepsilon>0$, we choose $F_{\varepsilon}:=\{\mu-\varepsilon \leq \varphi<\mu\}$, which has positive measure and is bounded. Moreover, by (3.4), $\rho=1$ on $F_{\varepsilon}$. For any 
$m>m^{\prime}>m-\left|F_{\varepsilon}\right|$, we consider $\tilde{\rho}=\rho-\left(\left(m-m^{\prime}\right) /\left|F_{\varepsilon}\right|\right) \chi_{F_{\varepsilon}}$ and bound, much as we did before,

$$
\begin{aligned}
E\left(m^{\prime}\right) & \leq \mathcal{E}[\tilde{\rho}]=E(m)-\frac{m-m^{\prime}}{\left|F_{\varepsilon}\right|} \int_{F_{\varepsilon}} \varphi(x) \mathrm{d} x+\left(\frac{m-m^{\prime}}{\left|F_{\varepsilon}\right|}\right)^{2} \mathcal{E}\left[\chi_{F_{\varepsilon}}\right] \\
& \leq E(m)-(\mu-\varepsilon)\left(m-m^{\prime}\right)+\left(\frac{m-m^{\prime}}{\left|F_{\varepsilon}\right|}\right)^{2} \mathcal{E}\left[\chi_{F_{\varepsilon}}\right] .
\end{aligned}
$$

This implies (note $m^{\prime}-m<0$ )

$$
\liminf _{m^{\prime} \uparrow m} \frac{E\left(m^{\prime}\right)-E(m)}{m^{\prime}-m} \geq \mu-\varepsilon,
$$

which proves the right inequality in the lemma.

Now assume that $\inf \varphi=\mu$. In this case, we simply choose $\tilde{\rho}=\left(m^{\prime} / m\right) \rho$ for any $m^{\prime}<m$, and obtain

$$
\begin{aligned}
E\left(m^{\prime}\right) & \leq \mathcal{E}[\tilde{\rho}] \leq\left(\frac{m^{\prime}}{m}\right)^{2} E(m)=E(m)-\frac{m^{2}-\left(m^{\prime}\right)^{2}}{2 m^{2}} \int_{\mathbb{R}^{d}} \rho \varphi \mathrm{d} x \\
& \leq E(m)-\frac{m^{2}-\left(m^{\prime}\right)^{2}}{2 m} \mu .
\end{aligned}
$$

That is,

$$
\frac{E\left(m^{\prime}\right)-E(m)}{m^{\prime}-m} \geq \frac{m+m^{\prime}}{2 m} \mu,
$$

which again implies the right inequality in the lemma.

\section{Diameter Bound}

It is known [5] that, even for the general interaction kernels of the previous section, the support of minimizers is bounded. An important ingredient in the proof of Theorems 1.1 and 1.2 is a quantitative version of this result which controls the size of the support in terms of $m$. More precisely, we show that the diameter of the support of $\rho$ grows at most like $m^{1 / 3}$ for large $m$. We emphasize that, while the results in this subsection can be extended to more general interaction kernels, for the sake of simplicity we restrict ourselves to the case $k(x)=|x|^{-1}+|x|^{\alpha}$.

Theorem 4.1. For any $\alpha>0$, there is a constant $C$ such that, for any $m>0$ and any minimizer $\rho$ of $E_{\alpha}(m)$, we have

$$
\operatorname{diam} \operatorname{supp} \rho \leq C \max \left\{1, m^{1 / 3}\right\} .
$$

The proof will rely on two auxiliary lemmas which we state and prove next. With any $\rho$ we associate its potential

$$
\varphi(x)=\int_{\mathbb{R}^{3}}\left(\frac{1}{|x-y|}+|x-y|^{\alpha}\right) \rho(y) \mathrm{d} y .
$$


The first lemma does not require $\rho$ to be a minimizer. In fact, this lemma can be used to prove the existence of a minimizer.

Lemma 4.2. For any $\rho \geq 0$ with $\int_{\mathbb{R}^{3}} \rho(y) \mathrm{d} y=m$, we have

$$
\sup _{a \in \mathbb{R}^{3}} \int_{B_{R}(a)} \rho(y) \mathrm{d} y \geq m-\frac{2 \mathcal{E}_{\alpha}[\rho]}{m R^{\alpha}} .
$$

Proof. We bound

$$
\begin{aligned}
\varphi(x) & \geq \int_{\mathbb{R}^{3} \backslash B_{R}(x)}|x-y|^{\alpha} \rho(y) \mathrm{d} y \geq R^{\alpha}\left(m-\int_{B_{R}(x)} \rho(y) \mathrm{d} y\right) \\
& \geq R^{\alpha}\left(m-\sup _{a \in \mathbb{R}^{3}} \int_{B_{R}(a)} \rho(y) \mathrm{d} y\right),
\end{aligned}
$$

and obtain

$$
\mathcal{E}_{\alpha}[\rho]=\frac{1}{2} \int_{\mathbb{R}^{3}} \rho(x) \varphi(x) \mathrm{d} x \geq \frac{m}{2} R^{\alpha}\left(m-\sup _{a \in \mathbb{R}^{3}} \int_{B_{R}(a)} \rho(y) \mathrm{d} y\right),
$$

which is the claimed inequality.

The second lemma provides an upper bound on the potential on the support of $\rho$. The method of proof is reminiscent of some arguments in geometric measure theory (see, e.g., [16, Lemma 4]).

Lemma 4.3. Let $\rho$ be a minimizer for $E_{\alpha}(m)$ for some $m>0$, and let $x \in \mathbb{R}^{3}$ be a Lebesgue point of $\rho$ with $\rho(x)>0$. Then,

$$
\varphi(x) \leq \frac{6+\alpha}{3} \frac{E_{\alpha}(m)}{m} .
$$

Proof. For fixed $r>0$ we define $\chi_{<}:=\chi_{B_{r}(x)}$ and $\chi_{>}=1-\chi_{<}$. We consider

$$
\tilde{\rho}(y):=\rho\left(\frac{y}{\ell_{r}}\right) \chi_{>}\left(\frac{y}{\ell_{r}}\right) \quad \text { with } \ell_{r}:=\left(\frac{m}{\int \chi_{>} \rho \mathrm{d} y}\right)^{1 / 3},
$$

which satisfies $\int_{\mathbb{R}^{3}} \tilde{\rho}(y) \mathrm{d} y=m$ and therefore, by optimality of $\rho$,

$$
\mathcal{E}_{\alpha}[\rho] \leq \mathcal{E}_{\alpha}[\tilde{\rho}]
$$

With the notation

$$
D^{(\beta)}[\sigma]:=\frac{1}{2} \iint_{\mathbb{R}^{3} \times \mathbb{R}^{3}} \sigma(y)\left|y-y^{\prime}\right|^{\beta} \sigma\left(y^{\prime}\right) \mathrm{d} y \mathrm{~d} y^{\prime},
$$




$$
\varphi^{(\beta)}(y):=\int_{\mathbb{R}^{3}}\left|y-y^{\prime}\right|^{\beta} \rho\left(y^{\prime}\right) \mathrm{d} y^{\prime},
$$

we have

$$
\begin{aligned}
\mathcal{E}_{\alpha}[\tilde{\rho}]= & \ell_{r}^{5} D^{(-1)}\left[\chi_{>} \rho\right]+\ell_{r}^{6+\alpha} D^{(\alpha)}\left[x_{>} \rho\right] \\
= & \ell_{r}^{5}\left(D^{(-1)}[\rho]-\int_{B_{r}(x)} \rho(y) \varphi^{(-1)}(y) \mathrm{d} y+D^{(-1)}\left[x_{<} \rho\right]\right) \\
& \quad+\ell_{r}^{6+\alpha}\left(D^{(\alpha)}[\rho]-\int_{B_{r}(x)} \rho(y) \varphi^{(\alpha)}(y) \mathrm{d} y+D^{(\alpha)}\left[x_{<} \rho\right]\right) .
\end{aligned}
$$

Therefore, (4.2) becomes

$$
\begin{gathered}
\ell_{r}^{5} \int_{B_{r}(x)} \rho(y) \varphi^{(-1)}(y) \mathrm{d} y+\ell_{r}^{6+\alpha} \int_{B_{r}(x)} \rho(y) \varphi^{(\alpha)}(y) \mathrm{d} y \\
\leq\left(\ell_{r}^{5}-1\right) D^{(-1)}[\rho]+\left(\ell_{r}^{6+\alpha}-1\right) D^{(\alpha)}[\rho] \\
+\ell_{r}^{5} D^{(-1)}\left[x_{<} \rho\right]+\ell_{r}^{6+\alpha} D^{(\alpha)}\left[x_{<} \rho\right] .
\end{gathered}
$$

Since $\ell_{r} \geq 1$, we can bound the left side from below by

$$
\begin{aligned}
& \ell_{r}^{5} \int_{B_{r}(x)} \rho(y) \varphi^{(-1)}(y) \mathrm{d} y+\ell_{r}^{6+\alpha} \int_{B_{r}(x)} \rho(y) \varphi^{(\alpha)}(y) \mathrm{d} y \\
& \geq \int_{B_{r}(x)} \rho(y) \varphi(y) \mathrm{d} y .
\end{aligned}
$$

On the other hand, since $\rho \leq 1$ we can bound the last two terms on the right side by

$$
\begin{aligned}
D^{(-1)}\left[\chi_{<} \rho\right] & \leq D^{(-1)}\left[\chi_{<}\right]=C_{1} r^{5}, \\
D^{(\alpha)}\left[\chi_{<} \rho\right] & \leq D^{(\alpha)}\left[\chi_{<}\right]=C_{2} r^{6+\alpha},
\end{aligned}
$$

for some constants $C_{1}$ and $C_{2}$. Therefore, we obtain

$$
\begin{aligned}
& \frac{1}{\left|B_{r}(x)\right|} \int_{B_{r}(x)} \rho(y) \varphi(y) d y \leq \\
& \quad \leq \frac{\ell_{r}^{5}-1}{\left|B_{r}(x)\right|} D^{(-1)}[\rho]+\frac{\ell_{r}^{6+\alpha}-1}{\left|B_{r}(x)\right|} D^{(\alpha)}[\rho]+C_{1}^{\prime} \ell_{r}^{5} r^{2}+C_{2}^{\prime} \ell_{r}^{6+\alpha} r^{3+\alpha} .
\end{aligned}
$$

We now want to let $r \rightarrow 0$. Since $x$ is a Lebesgue point of $\rho$ and since $\varphi$ is continuous, we have

$$
\lim _{r \rightarrow 0} \frac{1}{\left|B_{r}(x)\right|} \int_{B_{r}(x)} \rho(y) \varphi(y) d y=\rho(x) \varphi(x) .
$$


On the other hand, we have

$$
\frac{\ell_{r}^{3}-1}{\left|B_{r}(x)\right|}=\frac{1}{m-\int_{B_{r}(x)} \rho(y) \mathrm{d} y} \frac{1}{\left|B_{r}(x)\right|} \int_{B_{r}(x)} \rho(y) \mathrm{d} y \rightarrow \frac{\rho(x)}{m} .
$$

Since

$$
\frac{\ell_{r}^{5}-1}{\ell_{r}^{3}-1} \rightarrow \frac{5}{3} \text { and } \quad \frac{\ell_{r}^{6+\alpha}-1}{\ell_{r}^{3}-1} \rightarrow \frac{6+\alpha}{3},
$$

we finally conclude that

$$
\rho(x) \varphi(x) \leq\left(\frac{5}{3} D^{(-1)}[\rho]+\frac{6+\alpha}{3} D^{(\alpha)}[\rho]\right) \frac{\rho(x)}{m} .
$$

Bounding $5 \leq 6+\alpha$ and recalling that $\rho(x) \neq 0$, we obtain the lemma.

Proof of Theorem 4.1. If we choose

$$
R=\left(\frac{4 E_{\alpha}(m)}{m^{2}}\right)^{1 / \alpha}
$$

and $\rho$ is a minimizer, then $m-2 \mathcal{E}_{\alpha}[\rho] /\left(m R^{\alpha}\right) \geq m / 2$, and therefore Lemma 4.2 (and the continuity of $\left.a \mapsto \int_{B_{R}(a)} \rho(y) \mathrm{d} y\right)$ implies there is an $a \in \mathbb{R}^{3}$ such that

$$
\int_{B_{R}(a)} \rho(y) \mathrm{d} y \geq \frac{m}{2} .
$$

From this we conclude that for any $x \in \mathbb{R}^{3}$ with $|x-a|>(\sigma+1) R$, where $\sigma>0$ is a parameter to be determined later,

$$
\begin{aligned}
\varphi(x) & \geq \int_{B_{R}(a)}|x-y|^{\alpha} \rho(y) \mathrm{d} y \geq(|x-a|-R)^{\alpha} \int_{B_{R}(a)} \rho(y) \mathrm{d} y \\
& \geq(|x-a|-R)^{\alpha} \frac{m}{2}>\sigma^{\alpha} R^{\alpha} \frac{m}{2}=\sigma^{\alpha} 2 \frac{E_{\alpha}(m)}{m} .
\end{aligned}
$$

We choose $\sigma=(1+\alpha / 6)^{1 / \alpha}$, so that $2 \sigma^{\alpha}=(6+\alpha) / 3$, and then Lemma 4.3 implies that $\rho(x)=0$ whenever $|x-a|>(\sigma+1) R$. Thus,

$$
\operatorname{diam} \operatorname{supp} \rho \leq 2(\sigma+1) R=2\left(\left(1+\frac{\alpha}{6}\right)^{1 / \alpha}+1\right)\left(4 \frac{E_{\alpha}(m)}{m^{2}}\right)^{1 / \alpha} .
$$

Finally, by computing with trial functions we obtain

$$
E_{\alpha}(m) \leq \mathcal{E}_{\alpha}\left[m \chi_{B_{(3 /(4 \pi))^{1 / 3}}}\right]=C_{1} m^{2} \quad \text { if } m \leq 1
$$


(the radius has not been optimized) and

$$
E_{\alpha}(m) \leq \mathcal{E}_{\alpha}\left[x_{B_{(3 m /(4 \pi))^{1 / 3}}}\right]=C_{2} m^{5 / 3}+C_{3} m^{2+\alpha / 3} \quad \text { if } m>0,
$$

which implies that $E_{\alpha}(m) / m^{2} \leq C_{4} \max \left\{1, m^{\alpha / 3}\right\}$. Inserting this into the diameter bound, we obtain the theorem.

The following consequence of Theorem 4.1 will be used in the proof of Theorems 1.1 and 1.2.

Corollary 4.4. Let $\rho$ be a minimizer for $E_{\alpha}(m)$ and $x \in \operatorname{supp} \rho$. Then, if $\alpha \leq 2$,

$$
\min \left\{m, m^{(\alpha+1) / 3}\right\} \lesssim \int_{\mathbb{R}^{3}}|x-y|^{\alpha-2} \rho(y) \mathrm{d} y \lesssim m^{(\alpha+1) / 3},
$$

and, if $\alpha \geq 2$,

$$
m^{(\alpha+1) / 3} \lesssim \int_{\mathbb{R}^{3}}|x-y|^{\alpha-2} \rho(y) \mathrm{d} y \lessgtr \max \left\{m, m^{(\alpha+1) / 3}\right\}
$$

with implicit constants depending only on $\alpha$.

Proof. By the "bathtub principle" [15, Theorem 1.14], since $0 \leq \rho \leq 1$ and $\int \rho \mathrm{d} y=m$, we have

$$
\begin{aligned}
\int_{\mathbb{R}^{3}}|x-y|^{\alpha-2} \rho(y) \mathrm{d} y & \leq \int_{B_{(3 m /(4 \pi))^{1 / 3}(x)}}|x-y|^{\alpha-2} \mathrm{~d} y \\
& =C m^{(\alpha+1) / 3} \text { if } \alpha \leq 2
\end{aligned}
$$

and

$$
\begin{aligned}
\int_{\mathbb{R}^{3}}|x-y|^{\alpha-2} \rho(y) \mathrm{d} y & \geq \int_{B_{(3 m /(4 \pi))^{1 / 3}(x)}}|x-y|^{\alpha-2} \mathrm{~d} y \\
& =C m^{(\alpha+1) / 3} \text { if } \alpha \geq 2 .
\end{aligned}
$$

(Note that these inequalities are valid for any $x \in \mathbb{R}^{3}$.)

We now prove the opposite inequalities. Let $d=C \max \left\{1, m^{1 / 3}\right\}$, where $C$ is the constant from Theorem 4.1, so that the support of $\rho$ is contained in a ball $B$ of radius $d$. We shall prove the inequalities in the corollary for every $x \in B$, and therefore, in particular, for all $x \in \operatorname{supp} \rho$. Note that for any $x \in B$, supp $\rho$ is contained in a ball of radius $2 d$ around $x$. Let $c:=2\left(1-3 m /\left(4 \pi(2 d)^{3}\right)\right)^{1 / 3}$, so that the spherical shell between radii $c d$ and $2 d$ has volume $m$. Then, again by the "bathtub principle," if $x \in B$,

$$
\begin{aligned}
\int_{\mathbb{R}^{3}}|x-y|^{\alpha-2} \rho(y) \mathrm{d} y & \geq \int_{B_{2 d}(x) \backslash B_{c d}(x)}|x-y|^{\alpha-2} \mathrm{~d} y \\
& =C^{\prime} d^{\alpha+1}\left(2^{\alpha+1}-c^{\alpha+1}\right) \quad \text { if } \alpha<2
\end{aligned}
$$


and

$$
\begin{aligned}
\int_{\mathbb{R}^{3}}|x-y|^{\alpha-2} \rho(y) \mathrm{d} y & \leq \int_{B_{2 d}(x) \backslash B_{c d}(x)}|x-y|^{\alpha-2} \mathrm{~d} y \\
& =C^{\prime} d^{\alpha+1}\left(2^{\alpha+1}-c^{\alpha+1}\right) \quad \text { if } \alpha>2 .
\end{aligned}
$$

To complete the proof of the corollary, we note that

$$
d^{\alpha+1}\left(2^{\alpha+1}-c^{\alpha+1}\right) \approx \begin{cases}m & \text { if } m \leq 1, \\ m^{(\alpha+1) / 3} & \text { if } m \geq 1,\end{cases}
$$

where $\approx$ means there are upper and lower bounds on the ratio with finite, positive constants depending only on $\alpha$.

5. Proof of the Main Results, Theorems 1.1 AND 1.2

5.1. Outline of the proof. Both proofs of Theorems 1.1 and 1.2 rely on Proposition 2.1, which implies that $\Delta \varphi=0$ on $\{\varphi=\mu\}$. To apply this proposition, we need to verify that $\varphi \in W_{\text {loc }}^{2,1}\left(\mathbb{R}^{3}\right)$; in the following lemma, we will show that in fact $\varphi \in W_{\text {loc }}^{2, p}\left(\mathbb{R}^{3}\right)$ for any $p<\infty$.

We recall that, given $\rho, \varphi$ is defined by (4.1).

Lemma 5.1. For any $\rho \in L^{\infty}$ with $|x|^{\alpha} \rho \in L^{1}\left(\mathbb{R}^{3}\right)$, one has $\varphi \in W_{\text {loc }}^{2, p}\left(\mathbb{R}^{3}\right)$.

Proof. It is easy to see that $\varphi$ is a continuous function, so it is enough to show that $\partial_{i} \partial_{j} \varphi \in L_{\mathrm{loc}}^{p}\left(\mathbb{R}^{3}\right)$ for any $i, j$. We decompose $\varphi=\varphi_{-1}+\varphi_{\alpha}$ as in the proof of Lemma 4.3. We have $-\Delta \varphi_{-1}=4 \pi \rho$ in the sense of distributions. Since $\rho \in L^{p}\left(\mathbb{R}^{3}\right)$ for any $p<\infty$, we deduce from the Calderon-Zygmund inequality (see, e.g., [13, Theorem 9.9]) that $\partial_{i} \partial_{j} \varphi_{-1} \in L^{p}\left(\mathbb{R}^{3}\right)$.

In fact, we give an elementary proof of the weaker fact that $\partial_{i} \partial_{j} \varphi_{-1} \in L^{2}\left(\mathbb{R}^{3}\right)$ (which, however, is sufficient for our application of Proposition 2.1). Because $\rho \in L^{2}\left(\mathbb{R}^{3}\right)$ and since the Fourier transform of $e^{-|x|} /|x|$ is a constant times $1 /\left(1+p^{2}\right)$, we deduce that $e^{-|x|} /|x| * \rho \in H^{2}\left(\mathbb{R}^{3}\right)$. Moreover, since

$$
\left|\partial_{i} \partial_{j} \frac{1-e^{-|x|}}{|x|}\right| \lesssim|x|^{-1}(1+|x|)^{-2}
$$

and $\rho \in L^{p}\left(\mathbb{R}^{3}\right)$ for $3<p<\infty$, we conclude that $\partial_{i} \partial_{j}\left(1-e^{-|x|}\right) /|x| * \rho \in L^{\infty}$.

For the $\varphi_{\alpha}$ piece we use the fact that $\left.\left.\left|\partial_{i} \partial_{j}\right| x\right|^{\alpha}|\leq C| x\right|^{\alpha-2}$. Using $\rho \in L^{\infty}$ and $|x|^{\alpha} \rho \in L^{1}$, we again deduce that $\partial_{i} \partial_{j} \varphi_{\alpha} \in L^{\infty}$.

5.2. The regime of small mass: proof of Theorem 1.1. Let us consider the minimization problem

$$
E_{\alpha}^{*}:=\inf \left\{\mathcal{E}[\rho]: \rho \geq 0, \int_{\mathbb{R}^{3}} \rho(x) \mathrm{d} x=1\right\} .
$$


We know from $[7,20]$ that a minimizer exists, and from [8] that any minimizer is bounded, so

$$
M^{*}(\alpha):=\sup \left\{\|\rho\|_{\infty}^{-1}: \rho \text { minimizer for } E_{\alpha}^{*}\right\}>0 .
$$

The following simple fact is essentially contained in [5, Proof of Theorem 1.1 (i)], but for the sake of completeness we provide a proof.

Lemma 5.2. If $m \leq M^{*}(\alpha)$, then $E_{\alpha}(m)=m^{2} E_{\alpha}^{*}$.

Proof. We begin by proving, for every $m>0$, that $E_{\alpha}(m) \geq m^{2} E *$. For any $\rho$ with $0 \leq \rho \leq 1$ and $\int_{\mathbb{R}^{3}} \rho \mathrm{d} x=m$ we can take $\rho / m$ as a trial state for the $E_{\alpha}^{*}$ problem and obtain, by homogeneity,

$$
E_{\alpha}^{*} \leq \mathcal{E}_{\alpha}\left[\frac{\rho}{m}\right]=\frac{\mathcal{E}_{\alpha}[\rho]}{m^{2}} .
$$

Taking the infimum over all such $\rho$, we obtain $E_{\alpha}^{*} \leq E_{\alpha}(m) / m^{2}$, which is the inequality $\geq$ in the lemma.

For the converse inequality, let $m<M^{*}(\alpha)$ and choose a minimizer $\rho_{*}$ for $E_{\alpha}^{*}$ with $\left\|\rho_{*}\right\|_{\infty}^{-1} \geq m$. Then, $m \rho_{*}$ is an admissible trial state for the $E_{\alpha}(m)$ problem, and we obtain, again by homogeneity,

$$
E_{\alpha}(m) \leq \mathcal{E}_{\alpha}\left[m \rho^{*}\right]=m^{2} \mathcal{E}_{\alpha}\left[\rho_{*}\right]=m^{2} E_{\alpha}^{*},
$$

which is the inequality $\leq$ in the lemma. The equality extends to $m=M^{*}(\alpha)$ by continuity.

Proof of Theorem 1.1. Let $m \leq M^{*}(\alpha)$ and let $\rho$ be a minimizer for $E_{\alpha}(m)$. We infer from Lemma 5.2 that $E_{\alpha}^{\prime}(m)=2 m E_{\alpha}^{*}$ (for $m=M^{*}(\alpha)$ this holds for the left derivative), and therefore, by Lemma 3.1, that $\mu=2 m E_{\alpha}^{*}$. Thus,

$$
\begin{aligned}
\mu m & =2 m^{2} E_{\alpha}^{*}=2 E_{\alpha}(m)=\int_{\mathbb{R}^{3}} \varphi(x) \rho(x) \mathrm{d} x \\
& =\mu \int_{\mathbb{R}^{3}} \rho(x) \mathrm{d} x+\int_{\mathbb{R}^{3}}(\varphi(x)-\mu) \rho(x) \mathrm{d} x \\
& =\mu m+\int_{\mathbb{R}^{3}}(\varphi(x)-\mu) \rho(x) \mathrm{d} x,
\end{aligned}
$$

that is,

$$
\int_{\mathbb{R}^{3}}(\varphi(x)-\mu) \rho(x) \mathrm{d} x=0 .
$$

According to the Euler equation (3.4), $\varphi \leq \mu$ almost everywhere on $\{\rho>0\}$. Therefore, we conclude that

$$
\varphi=\mu \quad \text { almost everywhere on }\{\rho>0\} .
$$


By Proposition 2.1 and Lemma 5.1 we deduce that

$$
-\Delta \varphi=0 \quad \text { almost everywhere on }\{\rho>0\} .
$$

On the other hand, by an explicit calculation, we have

$$
-\Delta \varphi(x)=4 \pi \rho(x)-\alpha(\alpha+1) \int_{\mathbb{R}^{3}}|x-y|^{\alpha-2} \rho(y) \mathrm{d} y .
$$

Using Corollary 4.4 we can bound, for almost every $x \in \mathbb{R}^{3}$ with $\rho(x)=1$,

$$
4 \pi \rho(x)-\alpha(\alpha+1) \int_{\mathbb{R}^{3}}|x-y|^{\alpha-2} \rho(y) \mathrm{d} y \geq 4 \pi-C \max \left\{m, m^{(\alpha+1) / 3}\right\}
$$

For $\alpha \leq 2$, the maximum can be replaced by $m^{(\alpha+1) / 3}$. In any case, if we have $|\{\rho=1\}|>0$, we learn that $4 \pi \leq C \max \left\{m, m^{(\alpha+1) / 3}\right\}$ from (5.1), (5.2), and (5.3), that is, $m \geq \max \left\{4 \pi / C,(4 \pi / C)^{3 /(\alpha+1)}\right\}$ (and also $m \geq(4 \pi / C)^{3 /(\alpha+1)}$ if $\alpha \leq 2)$. This proves the theorem.

5.3. The regime of large mass: proof of Theorem 1.2. According to the Euler equation (3.4), $\varphi=\mu$ almost everywhere on $\{0<\rho<1\}$, and so again by Proposition 2.1 and Lemma 5.1 we deduce that

$$
-\Delta \varphi=0 \quad \text { almost everywhere on }\{0<\rho<1\} .
$$

As in the previous proof, we will compare this with the formula for $-\Delta \varphi$ from (5.2). Using Corollary 4.4, we can bound, for almost every $x \in \mathbb{R}^{3}$ with $0<\rho(x)<1$,

$$
4 \pi \rho(x)-\alpha(\alpha+1) \int_{\mathbb{R}^{3}}|x-y|^{\alpha-2} \rho(y) \mathrm{d} y \leq 4 \pi-C \min \left\{m, m^{(\alpha+1) / 3}\right\} .
$$

For $\alpha \geq 2$ the minimum can be replaced by $m^{(\alpha+1) / 3}$. In any case, we deduce from (5.4), (5.2) and (5.5) that $4 \pi \geq C \min \left\{m, m^{(\alpha+1) / 3}\right\}$, if $|\{0<\rho<1\}|>0$; that is, $m \leq \max \left\{4 \pi / C,(4 \pi / C)^{3 /(\alpha+1)}\right\}$ (and $m \geq(4 \pi / C)^{3 /(\alpha+1)}$ if $\alpha \geq 2$ ). This proves the theorem.

5.4. Extension to more general kernels. In this subsection we consider the minimization problem (3.3) with the energy function (3.1) involving a general kernel $k$ satisfying (3.2). 
Proposition 5.3. Assume that the distributional Laplacian $\Delta k$ is bounded from below by a locally integrable function and satisfies $\lim \inf _{|x| \rightarrow \infty} \Delta k>0$. Then, there exists an $m_{*}<\infty$ such that, for $m>m_{*}$, any minimizer $\rho$ for $E(m)$ satisfies $|\{\rho=1\}|=m$.

Proof. We argue as in the proof of Theorem 1.2, and therefore it suffices to show that $\Delta \varphi>0$ if $m$ is large enough. By assumption, there is a $\kappa \in L_{\text {loc }}^{1}\left(\mathbb{R}^{3}\right)$ and constants $c>0$ and $R>0$ such that $\Delta k \geq \kappa$ and $\kappa(x) \geq c$ if $|x| \geq R$. Thus,

$$
\begin{aligned}
\Delta \varphi(x) \geq & \int_{\mathbb{R}^{3}} \kappa(x-y) \rho(y) d y \geq-\int_{\{|x-y|<R\}} \kappa_{-}(x-y) \rho(y) d y \\
& +c \int_{\{|x-y| \geq R\}} \rho(y) d y \\
\geq- & \int_{\{|z|<R\}} \kappa_{-}(z) d z+c\left(m-(4 \pi / 3) R^{3}\right) .
\end{aligned}
$$

The right side is clearly positive if $m$ is large enough.

This proposition applies to, for instance, $k(x)=|x|^{-\beta}+|x|^{\alpha}$ with $0<\beta<1$ and $\alpha>2$. The same result holds also for $0<\alpha<2$, with a proof that is parallel to that of Theorem 1.2; as in that case, one needs in addition a bound on the diameter of the support of $\rho$. Such a bound can be obtained in the same way as in Section 4, and we omit the details.

\section{Appendix A. A Theorem About Subharmonic Functions. I}

In this appendix, we complement Corollary 2.2 by a similar result under different assumptions, which is interesting in its own right. While Corollary 2.2 concerns Sobolev functions and has a relatively straightforward proof, we now discuss the case of continuous functions without any integrability assumptions on derivatives. The proof is technically significantly more difficult, and we are greatly indebted to Luis Silvestre for showing us how to extend the result from $C^{1,1}$ functions to continuous functions. A further generalization will be discussed in the following appendix.

We recall that, if $u$ is a continuous function on an open set $\Omega$, we say that $-\Delta u \leq f$ in $\Omega$ in viscosity sense if, for any $x \in \Omega$ and any $\varphi \in C^{2}(\Omega)$ for which $u-\varphi$ has a local maximum at $x$, one has $-\Delta \varphi(x) \leq f(x)$.

Proposition A.1. Let $\Omega \subset \mathbb{R}^{d}, d \geq 1$, be an open set, and assume $u \in C(\Omega)$ satisfies $-\Delta u \leq-\varepsilon$ in $\Omega$ in viscosity sense for some $\varepsilon>0$. Then,

$$
|\{x \in \Omega: u(x)=\tau\}|=0 \quad \text { for any } \tau \in \mathbb{R} .
$$

This proposition has an elementary proof under the additional assumption $u \in C^{1,1}(\Omega)$, but remarkably it also holds without this assumption. We also note 
that the statement is wrong if we only assume $-\Delta u \leq 0$ in $\Omega$, as the example $u(x)=\max \left\{x_{1}, 0\right\}$ shows.

For the proof, we define for any set $\Omega \subset \mathbb{R}^{d}$ and any $u \in C(\Omega)$

$$
\begin{aligned}
& \bar{\Theta}(u, \Omega)(x):=\inf \left\{A \geq 0: \text { there is a } p \in \mathbb{R}^{d} \text { such that for all } y \in \Omega\right. \text {, } \\
& \left.u(y) \leq u(x)+p \cdot(y-x)+\frac{A}{2}|y-x|^{2}\right\}
\end{aligned}
$$

with the convention that $\inf \varnothing=+\infty$. We shall use the following deep result [6, Chapter 7] (see also [3, Proposition 3.1]).

Lemma A.2. Let $B \subset \mathbb{R}^{d}$ be a ball and $B^{\prime}$ a concentric ball with twice the radius. If $u \in C\left(B^{\prime}\right)$ satisfies $-\Delta u \leq 0$ in $B^{\prime}$ in viscosity sense, then

$$
\lim _{t \rightarrow \infty}\left|\left\{x \in B: \bar{\Theta}\left(u, B^{\prime}\right)(x)>t\right\}\right|=0 .
$$

In fact, there are bounds on how fast the measure of the set in the lemma tends to zero, but they are not important for us.

Proof. Replacing $u$ by $u-\tau$, we may assume that $\tau=0$. We will show that for every ball $B$ such that $\overline{B^{\prime}} \subset \Omega$ (where $B^{\prime}$ denotes the concentric ball with twice the radius), one has $|\{u=0\} \cap B|=0$. This will clearly imply the result.

We argue by contradiction and assume that $|\{u=0\} \cap B|>0$. According to Lemma A.2, we can choose $t$ so large that $\left|\left\{\bar{\Theta}\left(u, B^{\prime}\right)>t\right\} \cap B\right|<|\{u=0\} \cap B|$. This implies that $\left\{\bar{\Theta}\left(u, B^{\prime}\right) \leq t\right\} \cap\{u=0\} \cap B$ has positive measure, and we choose $x$ to be a Lebesgue point of this set and assume, after a translation, that $x=0$. Thus, we have $u(0)=0$, and there is a $p \in \mathbb{R}^{d}$ such that

$$
u(y) \leq p \cdot y+\frac{t}{2}|y|^{2} \quad \text { for all } y \in B^{\prime} .
$$

Let $B_{r}=\{y:|y|<r\}$. Since 0 is a Lebesgue point of $\{u=0\} \cap B$, we have $\left|B_{r}\right|^{-1}\left|\{u=0\} \cap B_{r}\right| \rightarrow 1$ as $r \rightarrow 0$, and therefore $p=0$. (Indeed, otherwise $u(y)$ would be negative in the cone $\left\{y \in \mathbb{R}^{d}: p \cdot y \leq-\delta|y|,|y|<2 \delta / t\right\}$ where $\delta<|p|$ is a fixed constant.) Therefore, we can bound, for every $r>0$ such that $B_{r} \subset B^{\prime}$,

$$
\int_{B_{r}} u(y) \mathrm{d} y \leq \int_{\{u \neq 0\} \cap B_{r}} u(y) \mathrm{d} y \leq \frac{t}{2} r^{2}\left|\{u \neq 0\} \cap B_{r}\right| .
$$

On the other hand, let us derive a lower bound on the left side. Using the Green's function for the ball, we find that for any $C^{2}$ function $v$ on $B_{\rho}$, one has

$$
v(0)=-c_{d} \int_{B_{\rho}}\left(\frac{1}{|y|^{d-2}}-\frac{1}{\rho^{d-2}}\right) \Delta v(y) \mathrm{d} y+\frac{(d-2) c_{d}}{\rho^{d-1}} \int_{|y|=\rho} v(y) \mathrm{d} \sigma(y),
$$


with $c_{d}=\left((d-2)\left|\mathbb{S}^{d-1}\right|\right)^{-1}$. (The formula for $d=1,2$ is similar and is omitted.) Multiplying by $\rho^{d-1}$ and integrating with respect to $\rho$, we obtain

$$
\begin{aligned}
& \left|B_{r}\right| v(0)= \\
& \quad-\frac{1}{d-2} \int_{B_{r}}\left(\frac{1}{d} \frac{r^{d}-|y|^{d}}{|y|^{d-2}}-\frac{1}{2}\left(r^{2}-|y|^{2}\right)\right) \Delta v(y) \mathrm{d} y+\int_{B_{r}} v(y) \mathrm{d} y .
\end{aligned}
$$

We apply this inequality to $v=\eta_{\delta} * u$, where $\eta_{\delta}(y)=\delta^{-d} \eta(y / \delta)$ with some mollifier $\eta \geq 0$, and note that $-\Delta v \leq-\varepsilon$. Using this inequality for the first term on the right side, and then letting $\delta \rightarrow 0$, we obtain, since $u(0)=0$,

$$
0 \leq-\frac{\varepsilon}{d-2} \int_{B_{r}}\left(\frac{1}{d} \frac{r^{d}-|y|^{d}}{|y|^{d-2}}-\frac{1}{2}\left(r^{2}-|y|^{2}\right)\right) \mathrm{d} y+\int_{B_{r}} u(y) \mathrm{d} y,
$$

that is,

$$
\int_{B_{r}} u(y) \mathrm{d} y \geq \varepsilon c_{d}^{\prime} r^{2}\left|B_{r}\right|,
$$

with a constant $c_{d}^{\prime}>0$ depending only on $d$.

Comparing (A.1) and (A.2), we find that for every $r>0$ such that $B_{r} \subset B^{\prime}$,

$$
\frac{\left|\{u \neq 0\} \cap B_{r}\right|}{\left|B_{r}\right|} \geq \frac{2 c_{d}^{\prime} \varepsilon}{t} .
$$

This contradicts the fact that 0 is a Lebesgue point of the set $\{u=0\} \cap B$. This proves the theorem.

\section{Appendix B. A Theorem About Subharmonic Functions. II}

In this appendix, we generalize Corollary 2.2 to general subharmonic functions. We learned the argument from Mikhail Sodin, to whom we are grateful.

2.1. Statement of the result and outline of the proof. If $u$ is a subharmonic function on an open set $\Omega \subset \mathbb{R}^{d}$, then the distribution $\Delta u$ is non-negative, and therefore extends to a non-negative, locally finite, regular Borel measure on $\Omega$, which we denote by $\mu_{u}$. (Here, regular means $\mu_{u}(A)=\inf \{\mu(O): O \supset A$ open $\}$ and $\mu_{u}(A)=\sup \{\mu(K): K \subset A$ compact $\}$ for every measurable set $A \subset \Omega$. Moreover, for us, measurable always means Borel measurable.)

Theorem B.1. Let $\Omega \subset \mathbb{R}^{d}$ be open and $u \in L_{\text {loc }}^{1}(\Omega)$ subharmonic such that $\mu_{u}(E)>0$ for any measurable $E \subset \Omega$ with $|E|>0$. Then, $|\{u=\tau\}|=0$ for any $\tau \in \mathbb{R}$.

The proof of this theorem hinges on the following two results. The first one extends an argument of Erëmenko-Sodin [10] (see also their references to earlier work by Øksendal) to arbitrary dimensions. 
Proposition B.2. Let $\Omega \subset \mathbb{R}^{d}$ be open and $u \in L_{\mathrm{loc}}^{1}(\Omega)$ be subharmonic and non-negative. Then,

$$
\mu_{u}\left(\left\{x \in \Omega: \lim _{r \rightarrow 0} \frac{\left|\left\{y \in B_{r}(x): u(y)=0\right\}\right|}{\left|B_{r}\right|}=1\right\}\right)=0 .
$$

In other words, $\Delta u$ vanishes on the set of Lebesgue points of $\{u=0\}$. In fact, our proof shows that there is an $\varepsilon_{d}>0$, depending only on $d$, such that

$$
\mu_{u}\left(\left\{x \in \Omega: \liminf _{r \rightarrow 0} \frac{\left|\left\{y \in B_{r}(x): u(y)=0\right\}\right|}{\left|B_{r}\right|}>1-\varepsilon_{d}\right\}\right)=0 .
$$

The second ingredient in our proof of Theorem B.1 is a special case of a lemma by Grishin (see [14] and also [21] and references therein).

Proposition B.3. Let $\Omega \subset \mathbb{R}^{d}$ be open, and let $u, v \in L_{\text {loc }}^{1}(\Omega)$ be subharmonic with $v \geq u$. Then, $\left.\left(\mu_{v}-\mu_{u}\right)\right|_{\{u=v>-\infty\}} \geq 0$.

Assuming these two propositions, we now show how they imply Theorem B.1.

Proof of Theorem B.1. Replacing $u$ by $u-\tau$, we may assume that $\tau=0$. The function $v:=u_{+}$is subharmonic, and, by setting

$$
E=\left\{x \in \Omega: \lim _{r \rightarrow 0} \frac{\left|\left\{y \in B_{r}(x): u(y) \leq 0\right\}\right|}{\left|B_{r}\right|}=1\right\},
$$

we deduce from Proposition B.2 that $\mu_{v}(E)=0$. Therefore, if

$$
E^{\prime}=\left\{x \in \Omega: \lim _{r \rightarrow 0} \frac{\left|\left\{y \in B_{r}(x): u(y)=0\right\}\right|}{\left|B_{r}\right|}=1\right\},
$$

then $E^{\prime} \subset E$ and therefore also $\mu_{v}\left(E^{\prime}\right)=0$. On the other hand, since $v \geq u$, Proposition B.3 implies that $\left.\left(\mu_{v}-\mu_{u}\right)\right|_{\{u \geq 0\}} \geq 0$. Therefore, if

$$
E^{\prime \prime}=\{x \in \Omega: u(x)=0\},
$$

then

$$
\mu_{u}\left(E^{\prime} \cap E^{\prime \prime}\right) \leq \mu_{v}\left(E^{\prime} \cap E^{\prime \prime}\right) \leq \mu_{v}\left(E^{\prime}\right)=0,
$$

so $\mu_{u}\left(E^{\prime} \cap E^{\prime \prime}\right)=0$. The assumed strict subharmonicity of $u$ therefore implies that $\left|E^{\prime} \cap E^{\prime \prime}\right|=0$. But, by Lebesgue's theorem, $\left|E^{\prime \prime} \backslash E^{\prime}\right|=0$, and therefore $\left|E^{\prime \prime}\right|=0$, as claimed.

2.2. Tools for the proof of the propositions. Thus, it remains only to prove Propositions B.2 and B.3. Their proofs rely on two ingredients. The first one is a variant of Green's formula, and the second one a general result about measures. For 
the first result, we therefore recall that Green's function for the unit ball $B \subset \mathbb{R}^{d}$ is given by

$$
\begin{aligned}
& G(x, y)= \\
& \begin{cases}\frac{1}{(d-2)\left|\mathbb{S}^{d-1}\right|}\left(\frac{1}{|x-y|^{d-2}}-\frac{1}{\left(1-2 x \cdot y+x^{2} y^{2}\right)^{(d-2) / 2}}\right) & \text { if } d \geq 3, \\
\frac{1}{2 \pi}\left(\ln \frac{1}{|x-y|}-\ln \frac{1}{\left(1-2 x \cdot y+x^{2} y^{2}\right)^{1 / 2}}\right) & \text { if } d=2 .\end{cases}
\end{aligned}
$$

Therefore, if $u$ is, say, $C^{2}$ in the unit ball, and continuous up to the boundary, then we have Green's representation formula

$$
u(x)=-\int_{B} G(x, y) \Delta u(y) \mathrm{d} y-\int_{\mathbb{S}^{d-1}} \frac{\partial G}{\partial v_{y}}(x, y) u(y) \mathrm{d} \sigma(y) .
$$

One consequence of this formula is that, if $u$ is subharmonic,

$$
\begin{aligned}
u(x) & \leq-\int_{\partial B} \frac{\partial G}{\partial v_{y}}(x, y) u(y) \mathrm{d} \sigma(y) \\
& =\frac{1}{\left|\mathbb{S}^{d-1}\right|} \int_{\mathbb{S}^{d-1}} \frac{1-x^{2}}{\left(1-2 x \cdot y+x^{2}\right)^{d / 2}} u(y) \mathrm{d} \sigma(y) .
\end{aligned}
$$

By using a simple density argument, based for instance on [15, Theorm 9.3], this inequality extends to any (not necessarily smooth) subharmonic function in the unit ball.

Next, we assume again that $u$ is, say, $C^{2}$ in $B$, and continuous on $\bar{B}$, and take $x=0$ in Green's representation formula. For $d \geq 3$, we obtain

$$
u(0)=-\frac{1}{(d-2)\left|\mathbb{S}^{d-1}\right|} \int_{B}\left(\frac{1}{|y|^{d-2}}-1\right) \Delta u(y) \mathrm{d} y+\frac{1}{\left|\mathbb{S}^{d-1}\right|} \int_{\mathbb{S}^{d-1}} u(y) \mathrm{d} \sigma(y) .
$$

We note that for any $\alpha>0$,

$$
\alpha^{-1}\left(|y|^{-\alpha}-1\right)=\alpha^{-1} \int_{0}^{\infty} \chi_{\left\{t<|y|^{-\alpha}-1\right\}} \mathrm{d} t=\int_{0}^{1} \chi_{\{|y|<\rho\}} \frac{\mathrm{d} \rho}{\rho^{\alpha+1}} .
$$

Thus,

$$
u(0)=-\frac{1}{\left|\mathbb{S}^{d-1}\right|} \int_{0}^{1} \mu_{u}\left(B_{\rho}\right) \frac{\mathrm{d} \rho}{\rho^{d-1}}+\frac{1}{\left|\mathbb{S}^{d-1}\right|} \int_{\mathbb{S}^{d-1}} u(y) \mathrm{d} \sigma(y) .
$$

This formula is also true if $d=2$. Moreover, by a density argument, based for instance on [15, Theorem 9.3], it extends to any function $u$ which is subharmonic in a neighborhood of the unit ball.

The second ingredient we use in the proof of the propositions is the following measure-theoretic result. 
Lemma B.4. Let $\mu$ be a signed, real, locally finite, regular Borel measure on $\mathbb{R}^{d}$, and let $E \subset \mathbb{R}^{d}$ be a measurable set such that, for any $x \in E$,

$$
\limsup _{r \rightarrow 0} \frac{\mu\left(B_{r}(x)\right)}{\left|B_{r}\right|} \geq 0 .
$$

Then, $\left.\mu\right|_{E} \geq 0$.

Proof of Lemma B.4. Let $A \subset E$ be measurable. We show that $\mu(A) \geq 0$. We may assume that $A$ is bounded. (Otherwise, we choose a tiling of $\mathbb{R}^{d}$ by half-open, disjoint cubes $Q_{j}$, and consider $A \cap Q_{j}$ for each fixed $j$.)

Let $\varepsilon>0$. By assumption, for any $x \in A$, there is a monotone decreasing sequence $\left(r_{n}(x)\right)_{n \in \mathbb{N}} \subset(0,1]$, tending to zero, such that $\mu\left(B_{r_{n}(x)}(x)\right) \geq$ $-\varepsilon\left|B_{r_{n}(x)}\right|$ for all $n$. We apply [18, Theorem 2.8], which is a consequence of the Besicovich covering theorem, to the family $\left\{\overline{B_{r_{n}}(x)}(x): x \in A, n \in \mathbb{N}\right\}$, and obtain a countable subfamily of disjoint balls $B_{j}$ such that $|\mu|\left(A \backslash \bigcup_{j} B_{j}\right)=0$. We write

$$
\mu(A)=\mu\left(\bigcup B_{j}\right)+\mu\left(A \backslash \bigcup_{j} B_{j}\right)
$$

and bound

$$
\mu\left(\bigcup B_{j}\right)=\sum_{j} \mu\left(B_{j}\right) \geq-\varepsilon \sum_{j}\left|B_{j}\right|=-\varepsilon\left|\bigcup_{j} B_{j}\right| \geq-\varepsilon C,
$$

where $C=\left|\left\{x \in \mathbb{R}^{d}: \operatorname{dist}(x, A) \leq 1\right\}\right|$, which is finite since $A$ is bounded. On the other hand,

$$
\left|\mu\left(A \backslash \bigcup_{j} B_{j}\right)\right| \leq|\mu|\left(A \backslash \bigcup_{j} B_{j}\right)=0,
$$

so $\mu(A) \geq-\varepsilon C$. As $\varepsilon>0$ is arbitrary, we conclude that $\mu(A) \geq 0$, as claimed.

2.3. Proof of the propositions. With these tools at hand we can now prove Propositions B.2 and B.3, and thereby complete the proof of Theorem B.1.

Proof of Proposition B.2. Let $\varepsilon_{d}=\left(1-2^{-d}\right) /\left(2^{d+2} 3\right)$ and

$$
E:=\left\{x \in \Omega: \liminf _{r \rightarrow 0} \frac{\left|\left\{y \in B_{r}(x): u(y)=0\right\}\right|}{\left|B_{r}\right|}>1-\varepsilon_{d}\right\} .
$$

We shall show that for any $x \in E$,

$$
\liminf _{r \rightarrow 0} \frac{\mu_{u}\left(B_{r}\right)}{\left|B_{r}\right|}=0 .
$$

This fact, together with Lemma B.4 (applied to $\mu=-\mu_{u}$ ), yields that $\left.\mu_{u}\right|_{E} \leq 0$. On the other hand, by subharmonicity $\mu_{u} \geq 0$, which implies that $\mu_{u}(E)=0$. 
(In fact, since one knows $\mu_{u} \geq 0$, the proof of Lemma B.4 can be somewhat abbreviated.)

Fix $x \in E$ and $\varepsilon \in\left(1-\liminf _{r \rightarrow 0}\left|\left\{y \in B_{r}(x): u(y)=0\right\}\right| /\left|B_{r}\right|, \varepsilon_{d}\right)$. We choose $r_{0}>0$ such that $\operatorname{dist}\left(x, \Omega^{c}\right)<r_{0}, M_{0}:=\sup _{|y-x|=r_{0}} u(x)<\infty$ and

$$
\left|\left\{y \in B_{r}(x): u(y) \neq 0\right\}\right| \leq \varepsilon\left|B_{r}\right| \quad \text { for all } 0<r \leq r_{0} .
$$

Moreover, let

$$
\theta(r)=\frac{\mathcal{H}^{d-1}\left(\left\{\omega \in \mathbb{S}^{d-1}: u(x+r \omega) \neq 0\right\}\right)}{\left|\mathbb{S}^{d-1}\right|} .
$$

We claim that, for any $0<r \leq r_{0}$, there exists an $r^{\prime} \in(r / 2, r)$ such that $\theta\left(r^{\prime}\right) \leq \varepsilon /\left(1-2^{-d}\right)=: \eta$. In fact, if we had $\theta(s)>\eta$ for all $s \in(r / 2, r)$, we would have

$$
\begin{aligned}
\varepsilon\left|B_{r}\right| & =\eta\left|\mathbb{S}^{d-1}\right| \int_{r / 2}^{r} s^{d-1} \mathrm{~d} s<\left|\mathbb{S}^{d-1}\right| \int_{r / 2}^{r} \theta(s) s^{d-1} \mathrm{~d} s \leq\left|\mathbb{S}^{d-1}\right| \int_{0}^{r} \theta(s) s^{d-1} \mathrm{~d} s \\
& =\left|\left\{y \in B_{r}(x): u(y) \neq 0\right\}\right| \leq \varepsilon\left|B_{r}\right|,
\end{aligned}
$$

a contradiction.

Applying this claim iteratively, we get a sequence $\left(r_{k}\right)$ with $\frac{1}{4} \leq r_{k+1} / r_{k} \leq \frac{1}{2}$ and $\theta\left(r_{k}\right) \leq \eta$. Let $M_{k}:=\sup _{|y-x|=r_{k}} u(x)$. Green's representation formula (B.2), together with the fact that $u(x) \geq 0$, implies that

$$
\int_{0}^{r_{k}} \mu_{u}\left(B_{\rho}(x)\right) \frac{\mathrm{d} \rho}{\rho^{d-1}} \leq \int_{\mathbb{S}^{d-1}} u\left(x+r_{k} \omega\right) \mathrm{d} \sigma(\omega) .
$$

Bounding the right side from above and the left side from below, we obtain

$$
\frac{2^{d-2}-1}{d-2} \mu_{u}\left(B_{r_{k} / 2}(x)\right) r_{k}^{-d+2}=\mu_{u}\left(B_{r_{k} / 2}(x)\right) \int_{r_{k} / 2}^{r_{k}} \frac{\mathrm{d} \rho}{\rho^{d-1}} \leq\left|\mathbb{S}^{d-1}\right| M_{k} .
$$

(If $d=2$, the constant $\left(2^{d-2}-1\right) /(d-2)$ is replaced by $\ln 2$.) This is the same as

$$
\frac{\mu_{u}\left(B_{r_{k} / 2}(x)\right)}{\left|B_{r_{k} / 2}\right|} \leq \frac{d-2}{2^{d-2}-1} d 2^{d} \frac{M_{k}}{r_{k}^{2}} .
$$

Thus,

$$
\liminf _{r \rightarrow 0} \frac{\mu_{r}\left(B_{r}\right)}{\left|B_{r}\right|} \leq \frac{d-2}{2^{d-2}-1} d 2^{d} \liminf _{k \rightarrow \infty} \frac{M_{k}}{r_{k}^{2}}
$$

and the proposition will follow if we can show that $M_{k} / r_{k}^{2} \rightarrow 0$ as $k \rightarrow \infty$. 
To show this, we observe that, after rescaling, inequality (B.1) implies that for any $y$ with $|y-x|=r_{k+1}$,

$$
u(y) \leq \frac{1}{\left|\mathbb{S}^{d-1}\right|} \int_{\mathbb{S}^{d-1}} \frac{r_{k}^{d-2}\left(r_{k}^{2}-r_{k+1}^{2}\right)}{\left(r_{k}^{2}-2 r_{k}(y-x) \cdot \omega+r_{k+1}^{2}\right)^{d / 2}} u\left(x+r_{k} \omega\right) \mathrm{d} \sigma(\omega) .
$$

Thus, recalling that $u \geq 0$,

$$
u(y) \leq \frac{r_{k}^{d-2}\left(r_{k}^{2}-r_{k+1}^{2}\right)}{\left(r_{k}-r_{k+1}\right)^{d}} \frac{1}{\left|\mathbb{S}^{d-1}\right|} \int_{\mathbb{S}^{d-1}} u\left(x+r_{k} \omega\right) \mathrm{d} \sigma(\omega) .
$$

We bound

$$
\frac{1}{\left|\mathbb{S}^{d-1}\right|} \int_{\mathbb{S}^{d-1}} u\left(x+r_{k} \omega\right) \mathrm{d} \omega \leq M_{k} \theta\left(r_{k}\right) \leq M_{k} \eta .
$$

Moreover, since $r_{k+1} / r_{k} \leq \frac{1}{2}$, we have

$$
\frac{r_{k}^{d-2}\left(r_{k}^{2}-r_{k+1}^{2}\right)}{\left(r_{k}-r_{k+1}\right)^{d}}=\frac{r_{k}^{d-2}\left(r_{k}+r_{k+1}\right)}{\left(r_{k}-r_{k+1}\right)^{d-1}} \leq \frac{3 / 2}{(1 / 2)^{d-1}}=2^{d-2} 3 .
$$

Since this bound is valid for any $y$ with $|y-x|=r_{k+1}$, we conclude that

$$
M_{k+1} \leq 2^{d-2} 3 \eta M_{k} .
$$

Since $r_{k+1} / r_{k} \geq \frac{1}{4}$, this implies that

$$
\frac{M_{k+1}}{r_{k+1}^{2}} \leq 2^{d-2} 3 \eta \frac{r_{k}^{2}}{r_{k+1}^{2}} \frac{M_{k}}{r_{k}^{2}} \leq 2^{d+2} 3 \eta \frac{M_{k}}{r_{k}^{2}}
$$

Iterating this, we obtain

$$
\frac{M_{k+1}}{r_{k+1}^{2}} \leq\left(2^{d+2} 3 \eta\right)^{k+1} \frac{M_{0}}{r_{0}^{2}}
$$

Since

$$
2^{d+2} 3 \eta=\frac{2^{d+2} 3 \varepsilon}{1-2^{-d}}<\frac{2^{d+2} 3 \varepsilon_{d}}{1-2^{-d}}=1,
$$

we infer that $M_{k+1} / r_{k+1}^{2} \rightarrow 0$ as $k \rightarrow \infty$, which concludes the proof.

Proof of Proposition B.3. Let $x \in \Omega$ such that $u(x)=v(x)>-\infty$.

We shall show there exists a sequence $\left(r_{n}\right) \subset(0, \infty)$, tending to zero, such that $\left(\mu_{v}-\mu_{u}\right)\left(B_{r_{n}}(x)\right) \geq 0$. According to Lemma B.4, this will imply that $\mu_{v}-\mu_{u} \geq 0$ on $\{u=v>-\infty\}$. 
For any $R<\operatorname{dist}\left(x, \Omega^{c}\right)$, we have, according to (B.2) and rescaling, $\int_{0}^{R}\left(\mu_{v}\left(B_{\rho}(x)\right)-\mu_{u}\left(B_{\rho}(x)\right)\right) \frac{\mathrm{d} \rho}{\rho^{d-1}}=\int_{\mathbb{S}^{d-1}}(v(x+R \omega)-u(x+R \omega)) \mathrm{d} \sigma(\omega)$.

Since the right side is non-negative by assumption, an $0<r_{0}<\operatorname{dist}\left(x, \Omega^{c}\right)$ exists such that $\mu_{v}\left(B_{r_{0}}(x)\right)-\mu_{u}\left(B_{r_{0}}(x)\right) \geq 0$. We now use the formula with $R$ replaced by $r_{0} / 2$. Again, the right side is non-negative, and so there is an $0<r_{1}<r_{0} / 2$ such that $\mu_{v}\left(B_{r_{1}}(x)\right)-\mu_{u}\left(B_{r_{1}}(x)\right) \geq 0$. Continuing in this way, we get a sequence with the claimed properties. This concludes the proof of the proposition.

Acknowledgements. The authors are grateful to Luis Silvestre and to Mikhail Sodin for showing us how to prove Proposition A.1 for continuous and for $L^{1}$ functions, respectively, to Almut Burchard and Paul Gauthier for their careful reading and many helpful comments, and to Haim Brézis for references related to Proposition 2.1. Support by U.S. National Science Foundation grants DMS1363432 (R.L.F.) and PHY-1265118 (E.H.L.) is acknowledged.

\section{REFERENCES}

[1] F. J. AlmgREN Jr. and E. H. LIEB, Symmetric decreasing rearrangement is sometimes continuous, J. Amer. Math. Soc. 2 (1989), no. 4, 683-773.

http://dx.doi.org/10.1090/S0894-0347-1989-1002633-4.MR1002633

[2] I. AOKI, A simulation study on the schooling mechanism in fish, NIPPON SUISAN GAKKAISHI [Bull. Jap. Soc. Scientific Fisheries] 48 (1982), no. 8, 1081-1088. http://dx.doi.org/10.2331/suisan.48.1081.

[3] S. N. Armstrong, L. E. Silvestre, and Ch. K. Smart, Partial regularity of solutions of fully nonlinear, uniformly elliptic equations, Comm. Pure Appl. Math. 65 (2012), no. 8, 1169-1184. http://dx.doi.org/10.1002/cpa.21394.MR2928094

[4] A. J. Bernoff and C. M. TOPAZ, A primer of swarm equilibria, SIAM J. Appl. Dyn. Syst. 10 (2011), no. 1, 212-250. http://dx.doi.org/10.1137/100804504.MR2788924

[5] A. BurChard, R. CHOKSI, and I. TOPAlOgLU, Nonlocal shape optimization via interactions of attractive and repulsive potentials, Indiana Math. J. 67 (2018), no. 1.

[6] L. A. CAfFarelli and X. CABrÉ, Fully Nonlinear Elliptic Equations, American Mathematical Society Colloquium Publications, vol. 43, American Mathematical Society, Providence, RI, 1995. http://dx.doi.org/10.1090/coll/043.MR1351007

[7] J. A. CAÑizo, J. A. CARRILlO, and F. S. PATACCHINi, Existence of compactly supported global minimisers for the interaction energy, Arch. Ration. Mech. Anal. 217 (2015), no. 3, 11971217.MR3356997

[8] J. A. Carrillo, M. G. Delgadino, and A. Mellet, Regularity of local minimizers of the interaction energy via obstacle problems, Comm. Math. Phys. 343 (2016), no. 3, 747-781. http://dx.doi.org/10.1007/s00220-016-2598-7.MR3488544

[9] R. CHOKSI, R. C. FETECAU, and I. TOPALOGLU, On minimizers of interaction functionals with competing attractive and repulsive potentials, Ann. Inst. H. Poincaré Anal. Non Linéaire 32 (2015), no. 6, 1283-1305. http://dx.doi.org/10.1016/j.anihpc.2014.09.004.MR3425263

[10] A. È. ERËMENKO and M. L. SODIN, A proof of the conditional Littlewood theorem on the distribution of the values of entire functions, Izv. Akad. Nauk SSSR Ser. Mat. 51 (1987), no. 2, 421-428, 448 (Russian); English transl., Math. USSR-Izv. 30 (1988), no. 2, 395-402. http://dx.doi.org/10.1070/IM1988v030n02ABEH001020.MR897006 
[11] R. C. FETECAU and Y. HUANG, Equilibria of biological aggregations with nonlocal repulsiveattractive interactions, Phys. D 260 (2013), 49-64. http://dx.doi.org/10.1016/j.physd.2012.11.004.MR3143993

[12] R. C. Fetecau, Y. Huang, and T. Kolokolnikov, Swarm dynamics and equilibria for a nonlocal aggregation model, Nonlinearity 24 (2011), no. 10, 2681-2716. http://dx.doi.org/10.1088/0951-7715/24/10/002.MR2834242

[13] D. Gilbarg and N. S. Trudinger, Elliptic Partial Differential Equations of Second Order, Classics in Mathematics, Springer-Verlag, Berlin, 2001. Reprint of the 1998 edition.MR1814364

[14] A. F. GRISHin, Sets of regular growth of entire functions. I, Teor. Funktsiu Funktsional. Anal. i Prilozhen. 40 (1983), 36-47 (Russian).MR738442

[15] E. H. Lieb and M. Loss, Analysis, 2nd ed., Graduate Studies in Mathematics, vol. 14, American Mathematical Society, Providence, RI, 2001. http://dx.doi.org/10.1090/gsm/014.MR1817225

[16] J. LU and F. ОТTO, Nonexistence of a minimizer for Thomas-Fermi-Dirac-von Weizsäcker model, Comm. Pure Appl. Math. 67 (2014), no. 10, 1605-1617.MR3251907

[17] M. MARCUS and V. J. MIZEL, Absolute continuity on tracks and mappings of Sobolev spaces, Arch. Rational Mech. Anal. 45 (1972), 294-320. http://dx.doi.org/10.1007/BF00251378.MR0338765

[18] P. MatTila, Geometry of Sets and Measures in Euclidean Spaces: Fractals and Rectifiability, Cambridge Studies in Advanced Mathematics, vol. 44, Cambridge University Press, Cambridge, 1995. http://dx.doi.org/10.1017/CB09780511623813.MR1333890

[19] J. SERRIN and D. E. VARBERG, A general chain rule for derivatives and the change of variables formula for the Lebesgue integral, Amer. Math. Monthly 76 (1969), no. 5, 514-520. http://dx.doi.org/10.2307/2316959.MR0247011

[20] R. SimiONE, D. SLEPČEV, and I. TOPALOGLU, Existence of ground states of nonlocal-interaction energies, J. Stat. Phys. 159 (2015), no. 4, 972-986.

http://dx.doi.org/10.1007/s10955-015-1215-z.MR3336989

[21] M. SODIN, Hahn decomposition for the Riesz charge of $\delta$-subharmonic functions, Math. Scand. 83 (1998), no. 2, 277-282. http://dx.doi.org/10.7146/math.scand.a-13856.MR1673934

RUPERT L. FRANK:

Mathematisches Institut

Ludwig-Maximilans Universität München

AND Department of Mathematics

Theresienstr. 39

80333 München, Germany

E-MAIL: rlfrank@caltech.edu

California Institute of Technology

1200 East California Boulevard

Pasadena, CA 91125, USA

ELLIOTT H. Lieb:

Departments of Mathematics and Physics

Princeton University

Fine Hall, Washington Road

Princeton, NJ 08544, USA

E-MAIL: lieb@princeton.edu

Received: August 1, 2016. 